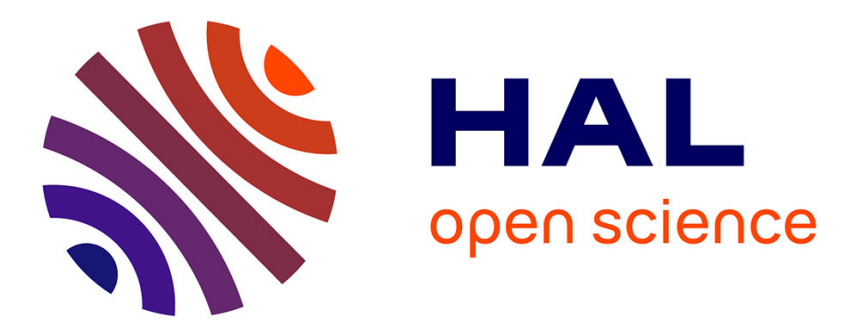

\title{
Local waiting time fluctuations along a randomly pinned crack front
}

Knut Jørgen Måløy, Stephane Santucci, Jean Schmittbuhl, Renaud Toussaint

\section{To cite this version:}

Knut Jørgen Måløy, Stephane Santucci, Jean Schmittbuhl, Renaud Toussaint. Local waiting time fluctuations along a randomly pinned crack front. Physical Review Letters, 2006, 96 (4), pp.045501. 10.1103/PhysRevLett.96.045501 . hal-00107346

\section{HAL Id: hal-00107346 \\ https://hal.science/hal-00107346}

Submitted on 29 Oct 2018

HAL is a multi-disciplinary open access archive for the deposit and dissemination of scientific research documents, whether they are published or not. The documents may come from teaching and research institutions in France or abroad, or from public or private research centers.
L'archive ouverte pluridisciplinaire $\mathbf{H A L}$, est destinée au dépôt et à la diffusion de documents scientifiques de niveau recherche, publiés ou non, émanant des établissements d'enseignement et de recherche français ou étrangers, des laboratoires publics ou privés. 


\title{
Local Waiting Time Fluctuations along a Randomly Pinned Crack Front
}

\author{
Knut Jørgen Måløy, ${ }^{1}$ Stéphane Santucci, ${ }^{1}$ Jean Schmittbuhl, ${ }^{2}$ and Renaud Toussaint ${ }^{2}$ \\ ${ }^{1}$ Fysisk Institutt, Universitetet i Oslo, P.O. Boks 1048 Blindern, N-0316 Oslo 3, Norway \\ ${ }^{2}$ Institut de Physique du Globe de Strasbourg, UMR 7516, 5 rue René Descartes, F-67084 Strasbourg Cedex, France
}

(Received 26 August 2005; published 30 January 2006)

\begin{abstract}
The propagation of an interfacial crack along a heterogeneous weak plane of a transparent Plexiglas block is followed using a high resolution fast camera. We show that the fracture front dynamics is governed by local and irregular avalanches with very large size and velocity fluctuations. We characterize the intermittent dynamics observed, i.e., the local pinnings and depinnings of the crack front by measuring the local waiting time fluctuations along the crack front during its propagation. The deduced local front line velocity distribution exhibits a power law behavior, $P(v) \propto v^{-\eta}$ with $\eta=2.55 \pm 0.15$, for velocities $v$ larger than the average front speed $\langle v\rangle$. The burst size distribution is also a power law, $P(S) \propto S^{-\gamma}$ with $\gamma=1.7 \pm 0.1$. Above a characteristic length scale of disorder $L_{d} \sim 15 \mu \mathrm{m}$, the avalanche clusters become anisotropic providing an estimate of the roughness exponent of the crack front line, $H=0.66$.
\end{abstract}

The physics community has recently paid a lot of attention to the study of damaging processes [1-3]. This interest is motivated not only by the practical benefits to many engineering domains, but also from a more fundamental point of view, by the diverse challenging questions brought forward, in particular, in statistical physics [4]. The role of heterogeneities during crack propagation is of central importance since they induce local pinnings of the crack front and subsequently trigger a very complex history of the fracture in the material. One of the consequences of this phenomenology is the roughness of fracture surfaces left by the crack. Indeed, cracks in heterogeneous media exhibit a self-affine morphology, with long range correlations. The associated roughness exponent was found to be very robust for different materials, over a broad range of length scales [5-11], and was further conjectured to be universal $[7,8]$. A recent work $[2,12]$ suggests that the origin of these self-affine long range correlations comes from the elastic interactions within the damage zone and proposes a link between the roughness exponent and the critical exponent $\nu$ for the correlation length of the damage clusters. More generally, front propagation in random media has become a challenging problem related to the dynamics of interfaces in many different physical systems theoretically connected, such as crack fronts [11], magnetic domain walls [13], or wetting contact lines [14-16], where elasticity and disorder compete to shape the interface.

In order to shed some light on the interactions between the crack front and material heterogeneities, a simplification to a two dimensional configuration - an interfacial crack - has been proposed both experimentally $[17,18]$ and theoretically [12,19]. The interfacial configuration provides a higher resolution since all locations of the crack front belong to the same plane. Moreover, using a transparent material and a high resolution fast camera, the detailed complex crack dynamics can be captured, following the crack front with a high precision both in time and space
[20]. So far experiments have been focused on the fracture front line morphology leading to the estimated roughness exponent $\zeta=0.55 \pm 0.03$ [17], followed up by a longer study showing $\zeta=0.63 \pm 0.03$ [18]. First attempts have been recently performed to analyze the interfacial crack front dynamics $[20,21]$. These studies have shown that the fracture front propagation is intermittent and can be described in terms of a Family-Vicsek scaling [22] with a roughness $\zeta=0.6$ and a dynamic exponent $\kappa=1.2 \pm 0.2$.

In this Letter, we study a system first studied experimentally by Schmittbuhl and Måløy [17,20]. Whereas previous studies focused on the morphology of the interfacial crack [17], we focus on the local crack dynamics, and on the distribution in both time and space of the waiting time during pinning events. To address this problem, we introduce a new analysis procedure in order to study the local waiting time fluctuations. The improved experimental techniques and resolution allow us to show that the dynamics of the fracture front is driven by local irregular avalanches with very large size and velocity fluctuations, and anisotropic shapes whose scaling is directly linked to the self-affine scaling of the crack front itself. This new set of experiments also confirms earlier results on such systems $[17,20]$.

We describe here experiments where two Plexiglas plates are annealed together to create a single block with a weak interface [17]. The plates are of dimensions: $32 \mathrm{~cm} \times 14 \mathrm{~cm} \times 1 \mathrm{~cm}$ and $34 \mathrm{~cm} \times 12 \mathrm{~cm} \times 0.4 \mathrm{~cm}$, and annealed together at $205^{\circ} \mathrm{C}$ under several bars of normal pressure. Before annealing, both plates are sandblasted on one side with $50 \mu \mathrm{m}$ steel particles or $100 \mu \mathrm{m}$ glass beads. Sandblasting introduces a random topography which induces local toughness fluctuations during the annealing procedure. We have measured the profile of a sandblasted Plexiglas surface, using a white light interferometry technique (performed at SINTEF-Oslo laboratory) and found that the local irregularities have a characteristic 
size about $15 \mu \mathrm{m}$ [23]. While the upper Plexiglas plate is clamped to a stiff aluminum frame, a press applies a normal displacement to the lower one $(1 \mathrm{~cm}$ thick) at a constant low speed which results in a stable crack propagation in mode I [17]. The fracture front is observed with a high resolution fast camera mounted on a microscope. Two different cameras have been used, a Kodak Motion Korder Analyzer CCD camera which records up to 500 frames per second (fps) with a $512 \times 240$ pixel resolution, and lately a much more powerful one, a Photron Ultima CMOS camera. Using this camera at a spatial resolution of $1024 \times$ 512 pixels, and an acquisition rate of $1000 \mathrm{fps}$ we can follow the stable crack front during more than $12 \mathrm{~s}$ (recording up to 12288 images). Different experiments have been performed varying the acquisition hardware, the microscope magnification corresponding to a pixel size between 1.7 to $10 \mu \mathrm{m}$, and the average front line speed ranging from 0.35 to $40 \mu \mathrm{m} / \mathrm{s}$. It is important to note that in all cases, the pixel size is smaller than the size of the local irregularities of about $20 \mu \mathrm{m}$ due to the sandblasting process.

In order to analyze the local waiting time fluctuations and the burst dynamics, we propose the following procedure: the fracture front lines extracted from image analysis of the digital pictures [see Fig. 1(a)] are added to obtain a waiting time matrix $\mathbf{W}(\mathbf{x}, \mathbf{y})$. This matrix has the dimension of the original image and an initial value equal to zero. We add the value 1 to the matrix element $w$ corresponding to each pixel of the detected front line position $(x, y)$. This procedure is performed for all frames of a given experiment in order to obtain the final waiting time matrix $\mathbf{W}(\mathbf{x}, \mathbf{y})$. A gray scale map of this matrix is shown in

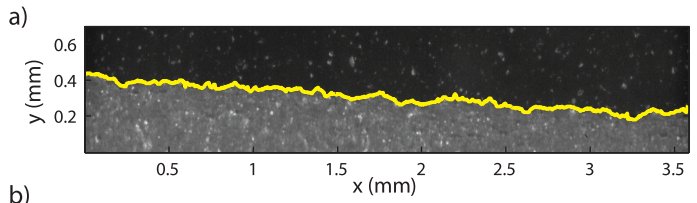

b)

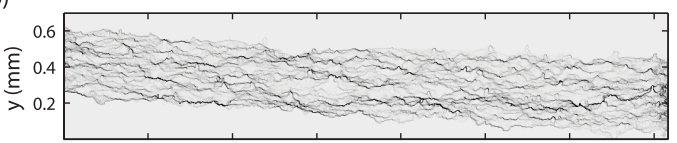

c)

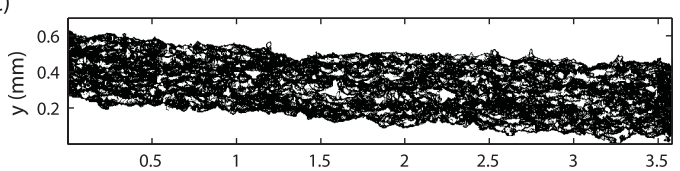

FIG. 1 (color). (a) Typical example of a picture recorded by the high speed camera (Photron Ultima) during an experiment with an average crack front speed $\langle v\rangle=28.1 \mu \mathrm{m} \mathrm{s}^{-1}$, and a pixel size $a=3.5 \mu \mathrm{m}$. The solid line represents the interface separating the uncracked (in black) and cracked parts extracted after image analysis. (b) Gray scale map of the waiting time matrix deduced from 10000 front positions recorded at a rate of 1000 fps. The darker parts show the longer waiting times. (c) Spatial distribution of clusters (in white) corresponding to velocities 10 times larger than the average crack front speed.
Fig. 1(b). The spatially random toughness along the weak interface generates a rough crack line in pinning the crack front [Fig. 1(a)], and triggers a rich burst activity on a wide range of length scales. The numerous and various regions of gray levels suggest this intermittent dynamics [Fig. 1(b)]. It is important to mention that the image recording is so fast that there are basically no holes in the waiting time matrix $\mathbf{W}(\mathbf{x}, \mathbf{y})$, i.e., no regions of zero values (apart from below the first front, above the last one, and a few artifacts due to impurities in the sample). Then, we can deduce from $\mathbf{W}(\mathbf{x}, \mathbf{y})$, a matrix $\mathbf{V}(\mathbf{x}, \mathbf{y})$ of the local normal speed of the interface at the time when the front went through a particular position, by computing the inverse value of the corresponding matrix element $w$ of $\mathbf{W}(\mathbf{x}, \mathbf{y})$ multiplied by the ratio of the pixel size $a$ and the typical time between two images $\delta t$. Therefore, we can associate to each pixel corresponding to the crack line in each image, a local front velocity $v=\frac{1}{w} \frac{a}{\delta t}$. Finally, we can obtain the probability distribution functions of the local waiting time $w$ and the local front velocity $v$ by estimating the occurrence number of each measured waiting time or velocity on all the pixels in all the fracture front line images. The velocity distribution $P(v /\langle v\rangle)$ is shown in Fig. 2 in a log-log scale. A data collapse is obtained for all different experimental conditions by scaling the local velocity $v$ with the average crack front speed $\langle v\rangle$ which varies from one experiment to another. A clear power law behavior of the velocity distribution $P(v /\langle v\rangle) \sim(v /\langle v\rangle)^{-\eta}$ is observed for velocities larger than $\langle v\rangle$ with a crossover to a slowly increasing function for velocities smaller than $\langle v\rangle$. A linear fit to the experimental data for $v /\langle v\rangle>1$ gives a slope $-\eta=-2.55 \pm 0.15$. The inset of the figure shows a double logarithmic plot of the corresponding waiting time distribution $P(w /\langle w\rangle)$, where $w$ is the waiting time, and $\langle w\rangle$ the average waiting time for each experiment. A linear

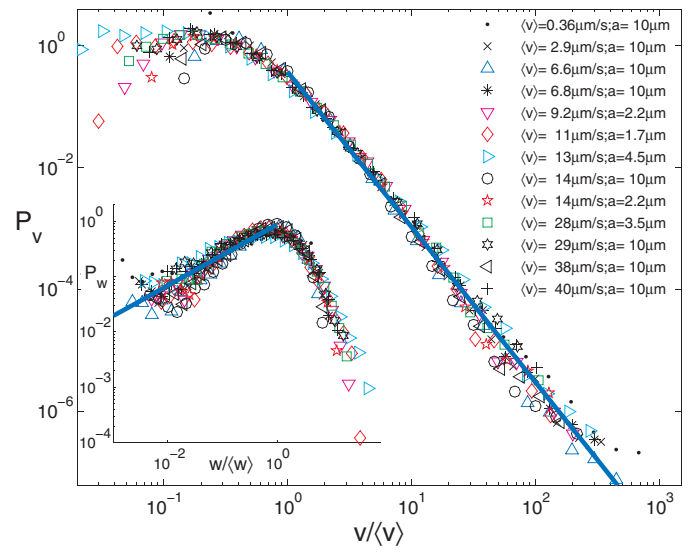

FIG. 2 (color). The velocity distribution $P(v /\langle v\rangle)$ as a function of the scaled velocity $v /\langle v\rangle$ for different experimental conditions (various average crack front speeds $\langle v\rangle$ and pixel sizes $a$ ). A fit (solid line) to all data for $v>\langle v\rangle$ has a slope -2.55 . Inset shows the corresponding waiting time distribution $P(w /\langle w\rangle)$ as a function of the scaled waiting time $w /\langle w\rangle$. The solid line represents a fit to all the data for $w<\langle w\rangle$ with a slope 0.55 . 
fit to the experimental data for $w<\langle w\rangle$ has a slope $0.55 \pm$ 0.15 , consistent with the exponent $\eta-2$ deduced from the velocity distribution. The power law distribution of the local velocities confirms once again previous observations, revealing a nontrivial underlying dynamics as observed on a fast video recording. It is important to note that even though the first moment of the velocity fluctuations $\langle v\rangle$ exists, the second and higher moments are ill defined and dominated by the largest velocity fluctuations. In an earlier work, the velocity distribution was investigated with a different method, based on the distance between subsequent fronts at a given time interval [20]. However, such a method proved out to produce results depending on the time interval chosen. Indeed, a short time between the front only gave contribution from the high velocity part of the distribution while a long time between the fronts gave a peak around the average velocity only. By using the concept of waiting time introduced in the present Letter, we are able to measure both high and low velocities. In the present case there exists a well defined length scale $a$ at which the velocity can be measured. Using different magnification of the microscope, we have checked the robustness of our procedure and shown the reproducibility of our results for different pixel sizes.

In order to analyze the local burst activity, we consider a thresholded matrix generated from the velocity matrix $\mathbf{V}(\mathbf{x}, \mathbf{y})$, by setting the matrix elements $v$ equal to one for $v>C\langle v\rangle$ and zero elsewhere, where $C$ is a constant of the order of a few unities. Figure 1(c) shows the spatial distribution of clusters of different sizes obtained from a thresholded matrix with a threshold level $C=10$. The white

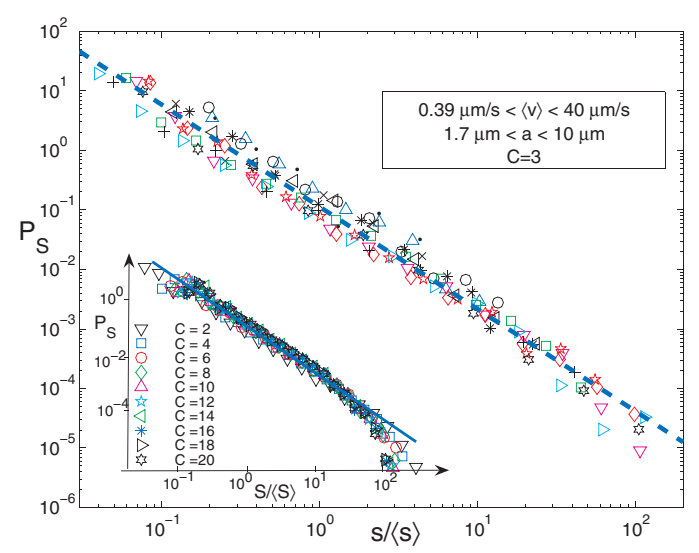

FIG. 3 (color). Burst size $S$ distribution $P(S /\langle S\rangle)$, normalized by the average burst size $\langle S\rangle$, for different experimental conditions (the various symbols correspond to those on Fig. 2). The bursts detected for each experiment correspond to clusters of velocities 3 times larger than the average crack front speed. A fit on all the data (dashed line) gives a slope equal to 1.71. Inset: Normalized bursts size distribution $P(S /\langle S\rangle)$ averaged over all the different experimental conditions, for a wide range of different threshold levels $C$. A fit to all the data, cutting the largest clusters at which a cutoff appears due to the lack of statistics (solid line), gives a slope equal to 1.67. clusters correspond to velocities 10 times larger than the average crack front speed $\langle v\rangle$. Then, we can extract from this thresholded velocity matrix the size distribution of the high velocity bursts. The clusters connected to the first and last front, and thus belonging to the upper and lower white parts are excluded from the analysis.

On Fig. 3, the cluster size distribution $P(S /\langle S\rangle)$ is shown for different experiments at a given threshold value $C=3$. First, we show a data collapse for all the different experiments performed by rescaling the clusters size $S$ with the average burst size $\langle S\rangle$. Moreover, we clearly observe that the burst size distribution $P(S /\langle S\rangle)$ follows a power law with an exponent $\gamma=1.7$ proving that the burst dynamics occurs on all length scales. We have checked that this critical behavior, and, in particular, the exponent $\gamma=$ $1.7 \pm 0.1$, is really robust: normalizing by the average burst size $\langle S\rangle$, we can rescale all the different distributions corresponding to diverse experimental conditions and a wide range of threshold level values $2<C<20$ (see inset of Fig. 3).

We expect a connection between the spatial scaling of the bursts, and the self-affine scaling of the front line itself on large scales. To investigate this, we have for each cluster $S$ chosen the smallest rectangular bounding box enclosing it. The size of the bounding box gives the length scale $L_{y}$ of the clusters along the growth direction and the length scale $L_{x}$ of the clusters along the average front line orientation. Figure 4 shows the dependence of the average size $\left\langle L_{y}\right\rangle$ on the length scale $\left\langle L_{x}\right\rangle$ in a double logarithmic plot, for a wide range of threshold values $2<C<20$, averaged over all the different experimental conditions (different pixels sizes and average crack front speeds). We clearly see that the avalanche clusters become anisotropic above a characteristic length scale $L_{d} \sim 15 \mu \mathrm{m}$. This typical size corre-

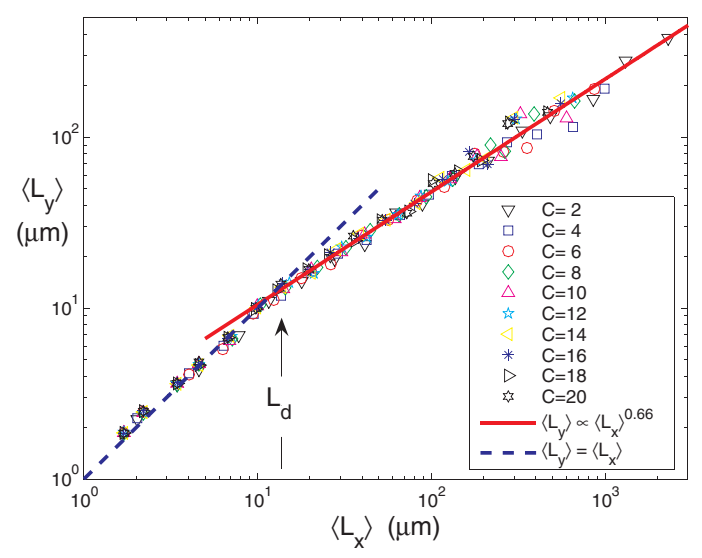

FIG. 4 (color). Average length scale $\left\langle L_{y}\right\rangle$ as function of the length scale $\left\langle L_{x}\right\rangle$, for different threshold levels $C$, averaged over all the different experiments performed. The solid line is a fit to the data points for $L_{x}>15 \mu \mathrm{m}$ and has a slope $H=0.66$, consistent with the roughness exponent of the fracture front line. The dotted line represents the curve $\left\langle L_{y}\right\rangle=\left\langle L_{x}\right\rangle$ and serves as a guide for the eye. 
sponds to the correlation length for the disorder introduced by the sandblasting technique [23]. Below $L_{d}$, the local toughness is marked by the same individual asperity and as a result the thresholded velocity bursts appear isotropic. A fit to the data points for $L_{x}>15 \mu \mathrm{m}$ gives a slope $H=$ 0.66 consistent with previous independent estimates of the roughness exponent $\zeta=0.63 \pm 0.03$ for the fracture front line. This result shows that the system exhibits self-affine scaling with the same roughness exponent $\zeta$ for the local burst as the fracture front line and brings a new confirmation of the roughness exponent found in our experiment, which is different and higher than most present theoretical or numerical predictions [19,24-26].

As mentioned in the introduction, the scaling behavior of elastic interfaces in random media is involved in various physical systems. Actually, the discrepancy between the theoretical and measured roughness exponent has also been reported recently, for contact lines of helium-4 [14] and water meniscus $[15,16]$ propagating on rough substrates. It confirmed that the Joanny-de Gennes model [27], usually proposed to describe the contact lines dynamics which leads to the same kind of equation of motion for crack fronts [28], is not sufficient [16]. Interestingly the roughness exponents found, respectively, equal to $0.56 \pm$ 0.03 and $0.52 \pm 0.04$, are close to the fracture front line roughness. Besides, for the helium- 4 meniscus, power law avalanche size distributions have been measured with exponents $\gamma$ from 0.99 to 1.3 , depending on the contact angle, which is different from the exponent $\gamma=1.7$ found in our experiments. However, avalanches are defined by Prevost et al. [14] based on subtraction between fronts, which is different from the present technique, and this $\gamma$ exponent could be sensitive to such a definition.

Recent simulations based on a quasistatic model and interpreted as a stress weighted percolation problem [12], give for the first time consistent results with the experimental roughness and dynamic exponent $[20,21]$. The insensitivity of the velocity distribution in our experiments on the average velocity of the front gives support to the quasistatic assumptions used in these simulations. However, the simulated process zone was not observed in our experiments above the micrometer scale. It should be mentioned that dynamical effects have also been introduced in a model [26] with a full elastodynamic description where elastic waves may trigger instabilities and modify the roughness of the crack front, leading to the value $\zeta=0.5$ [29].

No theory or simulations so far have investigated the local velocity or the burst fluctuations. It will be of great interest to perform these analyses on the numerical models for a direct comparison with our experimental work. This will hopefully clarify the importance of dynamical effects in modeling the fracture front propagation.

We thank A. Hansen, E. G. Flekkøy, and J. P. Vilotte for fruitful discussions, and E. L. Hinrichsen at SINTEF-Oslo for his hospitality. This work was supported by the CNRS/
NFR PICS program, the NFR Petromax and SUP program, and the french programs ACI "RNCC" and ACI "ALEAS."

[1] F. Célarié, S. Prades, D. Bonamy, L. Ferrero, E. Bouchaud, C. Guillot, and C. Marlière, Phys. Rev. Lett. 90, 075504 (2003).

[2] A. Hansen and J. Schmittbuhl, Phys. Rev. Lett. 90, 045504 (2003).

[3] I. Afek, E. Bouchbinder, E. Katzav, J. Mathiesen, and I. Procaccia Phys. Rev. E 71, 066127 (2005).

[4] H. J. Herrmann and S. Roux, Statistical Models for Fracture of Disordered Media (Elsevier, Amsterdam, 1990).

[5] B. B. Mandelbrot, D. E. Passoja, and A. J. Paullay, Nature (London) 308, 721 (1984);

[6] S. R. Brown and C. H. Scholz, J. Geophys. Res. 90, 12575 (1985).

[7] E. Bouchaud, G. Lapasset, and J. Planés, Europhys. Lett. 13, 73 (1990).

[8] K. J. Måløy, A. Hansen, E. L. Hinrichsen, and S. Roux, Phys. Rev. Lett. 68, 213 (1992).

[9] J. Schmittbuhl, S. Gentier, and S. Roux, Geophys. Res. Lett. 20, 639 (1993).

[10] J. Schmittbuhl, F. Schmitt, and C. H. Scholz, J. Geophys. Res. 100, 5953 (1995).

[11] E. Bouchaud, J. Phys. Condens. Matter 9, 4319 (1997).

[12] J. Schmittbuhl, A. Hansen, and G. Batrouni, Phys. Rev. Lett. 90, 045505 (2003).

[13] S. Lemerle et al., Phys. Rev. Lett. 80, 849 (1998).

[14] A. Prevost, E. Rolley, and C. Guthmann, Phys. Rev. B 65, 064517 (2002).

[15] S. Moulinet, C. Guthmann, and E. Rolley, Eur. Phys. J. E 8, 437 (2002).

[16] S. Moulinet, A. Rosso, W. Krauth, and E. Rolley, Phys. Rev. E 69, 035103(R) (2004).

[17] J. Schmittbuhl and K. J. Måløy, Phys. Rev. Lett. 78, 3888 (1997).

[18] A. Delaplace, J. Schmittbuhl, and K. J. Måløy, Phys. Rev. E 60, 1337 (1999).

[19] J. Schmittbuhl, S. Roux, J.P. Vilotte, and K. J. Måløy, Phys. Rev. Lett. 74, 1787 (1995).

[20] K. J. Måløy and J. Schmittbuhl, Phys. Rev. Lett. 87, 105502 (2001).

[21] S. Santucci, K. J. Måløy, R. Toussaint, and J. Schmittbuhl, NATO ASI Geilo Proceedings (to be published).

[22] F. Family and T. Vicsek, J. Phys. A 18, L75 (1985).

[23] S. Santucci, K. J. Måløy, R. Toussaint, and J. Schmittbuhl (to be published).

[24] J. P. Bouchaud, E. Bouchaud, G. Lapaset, and J. Planes, Phys. Rev. Lett. 71, 2240 (1993).

[25] S. Roux and A. Hansen, J. Phys. I 4, 515 (1994).

[26] S. Ramanathan and D. Fisher, Phys. Rev. B 58, 6026 (1998).

[27] J.F. Joanny and P. G. de Gennes, J. Chem. Phys.81, 552 (1984).

[28] H. Gao and J. R. Rice, J. Appl. Mech. 56, 828 (1989).

[29] E. Bouchaud, J. P. Bouchaud, D. S. Fisher, S. Ramanathan, and J. R. Rice, J. Mech. Phys. Solids 50, 1703 (2002). 